$\underline{\text { Research Article }}$

\title{
Group B $\beta$ haemolytic streptococcus colonization in pregnancy
}

\author{
B.N Dissanayake ${ }^{1}$, G.C Herath ${ }^{2}$, T.M Gamage ${ }^{1}$ \\ Sri Lankan Journal of Infectious Diseases 2015 Vol. 5(1):13-18 \\ DOI: http://dx.doi.org/10.4038/sljid.v5i1.7918
}

\begin{abstract}
Introduction: Group B $\beta$ haemolytic streptococcus (GBS) is a colonizer of the female genital tract and a known cause of neonatal infections. Identification of GBS colonization in pregnancy is the key to prevent such infections. Published data regarding GBS colonization in pregnancy are limited in Sri Lanka.
\end{abstract}

Objectives: The objectives of this study were to determine the prevalence of GBS colonization in pregnancy, to compare vaginal and rectal colonization rates and to find out the antibiotic susceptibility of the GBS isolates.

Methods: Lower vaginal and rectal swabs were collected separately from 100 pregnant women of 35 to 37 weeks gestation attending the obstetric clinics at Teaching Hospital Peradeniya from August to November 2011. Vaginal and rectal swabs were separately enriched using Todd Hewitt broth supplemented with gentamicin and nalidixic acid and incubated at $35-37{ }^{\circ} \mathrm{C}$. Following overnight incubation, the enriched broth was subcultured onto blood agar. Suspected colonies were identified with Gram stain, catalase and Lancefield's grouping. Susceptibility testing was performed using the Stokes method.

Results: GBS colonization in the study sample was found to be $30 \%$. GBS were recovered from both vaginal and rectal swabs in $20 \%$. It was isolated only from vaginal swabs in $4 \%$ and only from rectal swabs in $6 \%$. Rectal GBS colonization was $26 \%$ and higher than the vaginal colonization rate (24\%). The sensitivity to penicillin was found to be $100 \%$ while the sensitivity to erythromycin and clindamycin were $63.3 \%$ and $30 \%$ respectively.

Conclusion: This study implies the need for routine GBS screening in pregnancy and the importance of collecting both vaginal and rectal swabs for GBS screening.

Key words: Group B streptococcus, colonization, pregnancy

\begin{tabular}{l}
${ }_{1}^{1}$ Department of Microbiology, Faculty of Medicine, University of Peradeniya, Sri Lanka \\
${ }^{2}$ Postgraduate Institute of Science, University of Peradeniya, Sri Lanka \\
\hline Address for correspondence: Dr. BN Dissanayake; Department of Microbiology, Faculty of Medicine, \\
University of Peradeniya, Sri Lanka. Telephone No+94718148755 Email - nilanthidnk@ gmail.com \\
\hline
\end{tabular}

Received 13 January 2015 and revised version accepted 4 March 2015 


\section{Introduction}

Group B $\beta$ haemolytic streptococcus (GBS) can be found in the lower gastrointestinal tract and the lower genitourinary tract as a coloniser. GBS causes invasive disease primarily in infants, pregnant or postpartum women and in older adults, with the highest incidence among young infants. ${ }^{1}$ GBS infections in neonates primarily occur when the organism ascends from the vagina to the amniotic cavity or when a neonate is exposed to a colonized maternal birth canal during delivery. ${ }^{2}$ GBS causes two types of infections in neonates, early onset disease which occur during the first week of life, generally within the first 24 - 48 hours after birth and late onset disease which occurs after the first six days of life. ${ }^{3}$ In addition, colonised females may get infections including urinary tract infections, endometritis or chorioamnionitis. ${ }^{4}$

Several factors which increase the risk of neonatal disease have been identified. Those include positive GBS carriage in the current pregnancy, identification of GBS in urine during the current pregnancy, premature delivery, maternal pyrexia during delivery, prolonged rupture of membranes, intra-amniotic infection, young maternal age, black race, low maternal levels of GBS-specific anticapsular antibody and having a previous infant with early-onset GBS disease.,

As a preventive measure of GBS disease in neonates, CDC recommends routine screening of pregnant mothers at 35 -37 weeks of gestation for GBS carriage and to consider intra partum prophylactic antibiotics for the colonized mothers. ${ }^{1}$ Antibiotic susceptibility is important in determining prophylaxis, especially when one is allergic to penicillin. It also helps in choice of antibiotic for treatment if the mother or the infant develops an infection with this organism.

Several studies have been conducted in different parts of the world to find out the GBS colonization rates in pregnancy. Studies done in Belgium and Brazil have found GBS colonization rates of $22 \%$ and $24 \%$ respectively while a study conducted in India in 2012 have found $2.52 \%$ colonization rate in mothers and $1.26 \%$ in neonates. ${ }^{4,6}$ A prospective study carried out in Colombo South Teaching Hospital, Sri Lanka with the participation of 60 pregnant mothers at 35-36 weeks of gestation found a colonization rate of $26.7 \%$. $^{7}$

Hence, this study was conducted to find out the prevalence of GBS colonization among pregnant mothers who attend an obstetric clinic at Teaching hospital, Peradeniya, Sri Lanka, to compare vaginal and rectal colonization rates and to find out the sensitivity of the isolates to selected antibiotics.

\section{Methodology}

This study is a prospective descriptive study which was carried out for a period of 03 months from August to November 2011 at the Obstetric Clinics at Teaching Hospital, Peradeniya. Laboratory testing was carried out in the Microbiology Laboratory, Faculty of Medicine University of Peradeniya.

Lower vaginal and rectal samples were collected separately from 100 pregnant mothers at 35 to 37 weeks of gestation after obtaining informed consent. The swabs were enriched 
separately in Todd Hewitt Broth containing gentamicin $(8 \mu \mathrm{g} / \mathrm{ml})$ and nalidixic acid (15 $\mu \mathrm{g} / \mathrm{ml}$ ) and tubes were incubated at $35-37{ }^{\circ} \mathrm{C}$ for $18-24$ hours. ${ }^{1}$ Following incubation, the broth was subcultured onto a quality controlled human blood agar plate and incubated overnight at $35-37{ }^{\circ} \mathrm{C}$. Plates with no growth were re-incubated for another 24 hours. Plates were read at $24 \mathrm{hrs}$ and at $48 \mathrm{hrs}$. The plates which did not grow $\beta$ haemolytic streptococcus after 48 hours were considered as negative for GBS. Plates with a growth were subjected to routine testing to identify GBS. ${ }^{1}$

Gram stain followed by catalase test was performed and Gram positive cocci which were catalase negative were subjected to Lancefield grouping using a commercially available Streptococcal grouping latex test kit (OXOID-U.K). ${ }^{8,9}$ Antibiotic sensitivity of GBS isolates were determined using the Stokes method. ${ }^{10}$ Quality controlled blood agar plates were used and Penicillin $(0.25 \mu \mathrm{g})$, Erythromycin $(10 \mu \mathrm{g})$, and Clindamycin $(2 \mu \mathrm{g})$ were tested. Isolates showing flattening of the zone of inhibition around the clindamycin disc towards the erythromycin disc were noted as having inducible resistance to clindamycin. The rest of the results were read and interpreted according to the Stokes method. ${ }^{10}$ The minimum inhibitory concentration (MIC) of the isolates that were of intermediate sensitivity to penicillin were determined using a macro broth dilution method. ${ }^{11}$

\section{Results}

In the study population, $30(30 \%)$ were found to be colonized with GBS either at both the vagina and rectum or only in one site. GBS were recovered from both the lower vaginal and rectal swabs in $20(20 \%)$. Lower vaginal swabs alone were positive for GBS in $4(4 \%)$ of the subjects while rectal swabs alone were positive in $6(6 \%)$. The lower vaginal colonization rate was $24 \%$ and the rectal colonization rate was $26 \%$ in the study population.

Of the 30 GBS isolates, $20(66.7 \%)$ were sensitive to penicillin, while 10 (33.3\%) showed intermediate sensitivity (IS) and none were resistant. All 10 isolates with intermediate sensitivity were found to have an MIC ranging from 0.008 and $0.032 \mu \mathrm{g} / \mathrm{ml}$. According to the CLSI guidelines of 2013, all $\beta$ haemolytic streptococci with penicillin MIC of $\leq 0.12 \mu \mathrm{g} / \mathrm{ml}$ can be considered as sensitive. ${ }^{12}$ Nineteen $(63.3 \%)$ isolates were sensitive to erythromycin while eight $(26.7 \%)$ were of intermediate sensitivity and three $(10 \%)$ were resistant. Of the total GBS, $19(63.3 \%)$ were resistant to clindamycin. Interestingly, inducible resistance was not detected.

\section{Discussion}

In our study population, the overall GBS colonization rate was $30 \%$. This finding is consistent with another study done in the Colombo South Teaching hospital, where of 60 pregnant mothers screened, 16 were positive for GBS $(26.7 \%)^{7}$

Studies in other countries have shown varied colonization rates among pregnant mothers. A study done in 2009 in Belgium with 100 pregnant mothers at 35 - 37 gestation period showed a GBS colonization rate of $22 \% .{ }^{4}$ According to a cross sectional study done in Brazil in 2010, GBS colonization rate was $25.4 \%$ in 405 pregnant mothers at 35 to 37 weeks of gestation. ${ }^{13}$ 
In the current study, both lower vaginal swabs and rectal swabs were collected and processed separately. The rectal colonization rate $(26 \%)$ was higher than the vaginal colonization rate (24\%). However, according to a study done by Fernandopulle et al in 2006, the vaginal GBS colonization rate was higher $(87.5 \%)$ than the rectal colonization rate $(43.7 \%){ }^{7}$ A study done by El Aila et al (2009) found that vaginal swabs alone and rectal swabs alone could identify only $50 \%$ and $82 \%$ of all GBS of colonized mothers when compared with the rectovaginal swabs. ${ }^{4}$ According to Marconi et al (2010), combined swab from vaginal introitus, upper lateral vaginal vault and rectal region yielded the maximum colonization rate of $47.5 \%$ whilst the lower vaginal swab alone gave a colonization rate of $3.9 \% .{ }^{13}$

Results of the current study are not compatible with what has been found by Fernandopulle et al (2006). As found out by this study and many other studies, it is important to include a rectal swab in addition to a vaginal swab for GBS screening in pregnancy to improve sensitivity. Using the same swab to sample rectum and vagina (rectovaginal swab) is also a possibility, but acceptance can be low among pregnant women due to the inconvenience of the procedure.

The antibiotic of choice for prophylaxis as well as for treatment of GBS is penicillin. Although $33.3 \%$ of our isolates were designated as of intermediate sensitivity to penicillin using Stokes method, this was not confirmed by the MIC which was lower than $0.12 \mu \mathrm{g} / \mathrm{ml}$ in all isolates. The same observation has been made in Tanzania in 2009 where $10 \%$ of GBS isolates found to be intermediately sensitive to penicillin by Stokes method identified as sensitive to penicillin by MIC testing. Therefore, further testing need to be considered when dealing with such results.

In our study, $19(63.3 \%)$ isolates were found to be sensitive to erythromycin and $8(26.7 \%)$ resistant while $19(63.3 \%)$ and $2(6.7 \%)$ isolates were found to be of intermediate sensitivity and resistant to clindamycin respectively. Studies done in many other countries have found a lower level of resistance to erythromycin in this group of subjects. A study done in Australia on invasive GBS isolates collected from 1982 to 2006 have found $6.4 \%$ resistance while a study done in Kuwait in 2007 and Tanzania in 2009 found resistance rates of $12.6 \%$ and 13\% respectively. ${ }^{14,15,16} \mathrm{~A}$ higher resistance rate $(22 \%)$ to erythromycin was reported by Hasenbein et al (2004). ${ }^{17}$ Resistance to clindamycin in the current study was low (6.7\%) although $63.3 \%$ of isolates were detected as having intermediate sensitivity. This is a significant finding and needs further testing as clindamycin is considered an alternative in patients who do not tolerate penicillin. Although determination of MIC would have been helpful, resource limitations prevented further investigations on these isolates.

\section{Conclusions}

One third of mothers in the study were colonised with Group B $\beta$ haemolytic streptococci in the last trimester of pregnancy. All the isolates recovered were sensitive to penicillin while a significant proportion (26.7\%) was resistant to erythromycin. A considerable number of isolates were intermediately susceptible to clindamycin, which needs further confirmation. 
Although the study population was limited to a single hospital and the study duration was limited to 3 months, the results of this study are in accordance with current practice in many countries and emphasize the need to introduce routine screening of pregnant mothers at 35-37 weeks of pregnancy for GBS.

The study results also emphasize the need for using both vaginal and rectal swabs when screening is undertaken.

Penicillin remains the choice for both treatment and prophylaxis in this group of subjects.

\section{References}

1. Verani JR, McGee L and Schrag SL. Prevention of Perinatal Group B Streptococcal disease revised guidelines from CDC. MMWR 2010; 59(RR10):1-32. No doi

2. Richard AH, Pamela CC and Bruce DF. Microbiology $\left(2^{\text {nd }}\right.$ ed). Lippincott Williams \& Wilkins., 2007.

3. Hathlahawatta HMKN, Samarage DK, Liyanage, GS Chandrasiri S. A retrospective study on Group B beta haemolytic Streptococcal sepsis in early infancy. Sri Lanka Journal of. Child Health 2009; 38(4):124-126. No doi

4. El Aila NA, Tency I, Claeys G, Saerens B, et al. Genotyping of Streptococcus agalactiae (Group B Streptococci) isolated from vaginal and rectal swabs of women at 35-37 weeks of pregnancy. BMC Infectious Diseases 2009; 9:1471-2334.

doi: http://dx.doi.org/10.1186/1471-2334-9-153

5. Daniels J, Gray J, Pattison H, et al. Rapid testing for Group B Streptococcus during labour: a test accuracy study with evaluation of acceptability and cost-effectiveness. Health Technology Assessment 2009; 13:42. doi : http://dx.doi.org/10.3310/hta13420

6. Kulkarni AA, Pawar SG, Dharmadhikari CA and Kulkarni RD. Colonization of pregnant women and their newborn infants with group B Streptococci. Indian Journal of Medical Microbiology 2001; 19:1-4. No doi

7. Fernandopulle RC, Fernando SN, Peellawattage MK, Malluwawadu GN. Proportion of Group B Streptococcus carriage and their antibiotic sensitivity in pregnant mothers attending an antenatal clinic. Sri Lanka Medical Association 2006; 119th Annual Scientific sessions : pp36. No doi

8. Cowan I and Steel KJ. Manual for the identification of medical bacteria ( ${ }^{\text {rd }}$ ed). Cambridge., 1999.

9. Coyle MB. Manual of antibiotic susceptibility testing $\left(1^{\text {st }}\right.$ ed). Washington: American Society of Microbiology., 2005.

10. Sri Lanka College of Microbiologists. Microbiology laboratory manual ( $1^{\text {st }}$ ed). 2001. Andrews JM. Determination of minimum inhibitory concentrations. Journal of Antimicrobial Chemotherapy 2001; 48(S1):5-16. doi: http://dx.doi.org/10.1093/jac/48.suppl_1.5

11. Clinical and Laboratory Standards Institute: Performance standards for antimicrobial susceptibility testing M100-S23., 2013

12. Marconi C, Rocchetti TT, Rall VLM, et al. Detection of Streptococcus agalactiae colonization in pregnant women by using combined swab cultures: cross-sectional prevalence study. Sao Paulo Medical Journal 2010; 128(2):60-2.

doi: http://dx.doi.org/10.1590/S1516-31802010000200003 
13. Garland SM, Cottrill E, Markowski L et al. Antimicrobial resistance in Group B Streptococcus: the Australian experience. The Journal of Medical Microbiology 2011; 60: 230-5.

14. Boswihi SS, Udo EE and Al-Sweih N,. Serotypes and antibiotic resistance in Group B streptococcus isolated from patients at the Maternity Hospital, Kuwait. Journal of Medical Microbiology 2012; 61:126-31.

doi: http://dx.doi.org/10.1099/jmm.0.035477-0

15. Joachim A, Matee MI, Massawe FA, Lyamuya EF. Maternal and neonatal colonisation of group B Streptococcus at Muhimbili National Hospital in Dar es Salaam, Tanzania: prevalence, risk factors and antimicrobial resistance. BMC Public Health 2009; 9:1471-2458. doi: http://dx.doi.org/10.1186/1471-2458-9-437

16. Heelan JS, Hasenbein ME, McAdam AJ.. Resistance of Group B Streptococcus to selected antibiotics, including Erythromycin and Clindamycin. Journal of Clinical Microbiology 2004; 42(3):1263-1264. doi: 10.1128/JCM.42.3.1263-1264.2004 